\title{
A class of globally stabilising controllers for the control of wave energy devices for potable water production
}

\author{
Angeliki Lekka, Matthew C. Turner and John V. Ringwood
}

\begin{abstract}
This paper provides a stability analysis for a system that captures wave energy in order to produce potable water. The system, introduced in [1], is a Wave Energy Converter (WEC) of the point-absorber type coupled to a hydraulic Power Take-Off (PTO) that converts wave energy into pressure. Previous work has used a partial state-feedback controller with integral action and feed-forward to provide good nominal control behaviour. Although open-loop stability was proven in [1], no guarantees of closed-loop stability were given; in this paper we provide such guarantees for a class of controllers, of which the controller proposed in [1] is a special case.
\end{abstract}

\section{INTRODUCTION}

Wave energy is a renewable and environmentally friendly source of energy. It has been of interest to industry as well as the academic community for some time due to its wide availability and its potential to be used for the production of electricity and/or potable water in many coastal parts of the world.

Wave energy is mainly used for two purposes: the production of electricity and the production of potable water. Although the majority of the academic community has focused on the electricity production problem, this paper focuses on the production of potable water from waves, through a desalination ${ }^{1}$ process called Reverse Osmosis (RO). Wavepowered desalination appears particularly attractive since both of the primary requirements, seawater and energy, are available at the same location. Moreover, the desalination of seawater can be of great importance in arid areas, in developing countries with coastlines and also contribute to the drinking water independence of islands around the world.

Although the first design of a device that captures energy from the waves, viz a Wave Energy Converter (WEC), dates back to the $18^{t h}$ century [2], there is not a universally adopted method of capturing wave energy, and many WEC configurations have been proposed. The main classes of WECs currently available, both academically and commercially, are so-called oscillating bodies, overtopping devices, floating bodies and multi-body devices. The interested reader is referred to [3] for a more extensive and detailed review

Angeliki Lekka and Matthew C. Turner are with the Department of Engineering, University of Leicester, University Road, LE1 7RH, UK. al192@le.ac.uk, mct6@le.ac.uk

John Ringwood is with the Department of Electronic Engineering, National University of Ireland (NUI) Maynooth, Maynooth, Co. Kildare, Ireland. john.ringwood@eeng.nuim. ie

${ }^{1}$ Desalination is the process of converting sea water to potable drinking water. of such devices. The type of WEC examined in this paper is a point-absorber heaving buoy, in which the main source of power is the vertical oscillation of the buoy.

\section{A. Control of WECs}

Without some form of active control, WECs are essentially passive devices which would perform inefficiently due to the varying behaviour of the sea. The main aim of an active control system, is to improve the efficiency of the devices and to enable them to function well in different sea states. Active control systems have various objectives, including the maximisation of energy extraction from the waves, the minimisation of damage to the device due to large waves and the coordination of WEC arrays [4]. By some margin, most of the literature concentrates on the control of WECs for power generation where a number of different techniques have been proposed. Among the first approaches for maximising power generation was the approach known as reactive or complex conjugate control [5], [6], [7], [8], which maximises energy capture by creating destructive interference between the properties of the point-absorber and the incident waves. An alternative approach, known as latching (also known as phase control), is a nonlinear control approach in which the point absorber is held for approximately one quarter of the incoming wave, allowing the force of the WEC to reach high levels, and then released [1], [4], [9]. Another approach is so-called passive loading [5] where the energy capture is not maximum, but the power flow is unidirectional. More recently, researchers have begun to use model predictive control (MPC) for WEC control, which allows constraints involved with the WEC (position, velocity or force constraints) to be incorporated directly into the controller design [10], [11], [12].

There is also interest in controlling WECs so that they can be used for potable water production. In this application, maintaining a constant pressure in the reverse osmosis (RO) unit is key, in order to ensure efficient desalination (higher pressures give better efficiency) and avoid damage to the RO membrane (as a result of excessively high pressures). However, maintaining a constant pressure may not allow complete freedom of PTO force specification, which is desirable to facilitate maximum power transfer from the waves to the device. A solution which allows both objectives to be followed involves the use of a recently proposed PTO system [13], which effectively couples the RO unit (or electrical power generation) from the PTO input side, where optimum 
power transfer is effected. While this topic has not been treated extensively in the literature, it has attracted significant attention recently [14], [15], [16].

One notable drawback with much of the current literature is that although researchers have been keen to propose new control schemes for WECs, the formal analysis of such schemes is, with a few exceptions, largely absent. In particular, it is necessary to examine the stability properties of these schemes, because the WECs are often nonlinear and also because some control schemes themselves have nonlinear elements. Although the physical properties of most wave energy devices may allow bounded-input-boundedoutput stability to be inferred, internal stability cannot be taken for granted and may not always be trivial to prove. Such analysis also has practical implications because closedloop internal stability will prevent limit cycles and other strange behaviour occurring in WECs, and thus lead to control schemes which, when implemented on real WECs, bestow safe and reliable operation in varying conditions [10].

This paper focuses on the stability properties of a generic class of pseudo-nonlinear dynamic inversion (NDI) controllers applicable to point-absorber type heaving WECs; a special case of which is considered in [14], [1], where a partial state feedback (proportional) controller with feedforward and integral gains was proposed. In [14], [1] it was shown that the controller performed well in high fidelity simulations, although a formal stability analysis was not carried out. In [14], it was shown that the system was openloop stable, but the proof was dependent on the numerical solution of a number of Lyapunov equations and did not exploit the structure of the WEC equations in the analysis. In addition, the analysis was open-loop and not closed-loop.

The paper is structured as follows: Section II describes the system under consideration, Section III describes the stability analysis of the controller used and finally Section IV summarises the findings of this paper and gives the conclusions.

\section{DESCRIPTION OF THE SYSTEM}

The type of WEC studied here consists of a generic floating body oscillating in heave (point absorber) and its parameters can be found in Table I. A floating body is said to be a point absorber when its horizontal dimensions are small compared to the length of the incident wave. The body of the WEC is coupled to a Power Take-Off unit (PTO), which converts captured wave energy into useful quantities (e.g. pressure and flow) and in this case a pressure of 60bar was required to ensure efficiency of the reverse-osmosis process. The system under consideration consists of three distinct parts; the excitation force, the mechanical model of the buoy itself, and the PTO subsystem.

\section{A. Excitation force}

The excitation force model represents the motion of the ocean waves that exert a force on the buoy, thereby inducing motion. From a certain perspective, the excitation force can be considered as a disturbance to the system and there are a number of different ways to represent it. Here a model for producing irregular waves, based on the Pierson-Moskowitz (PM) [17] energy spectrum for fully developed seas, has been used and it is representative of a large number of ocean locations. The PM energy spectrum is given by

$$
E_{\omega}(\omega)=\frac{0.11 H_{\frac{1}{3}}^{2} T_{1}}{2 \pi}\left(\frac{\omega T_{1}}{2 \pi}\right)^{-5} e^{\left[-0.44\left(\frac{\omega T_{1}}{2 \pi}\right)^{-4}\right]}
$$

where $H_{\frac{1}{3}}$ is the significant wave height, $T_{1}$ is the mean wave period and $\omega$ is the wave frequency.

The time series corresponding to this spectrum can be calculated by dividing it into 100 equally spaced frequencies at intervals of $\Delta \omega=0.0325 \mathrm{rad} / \mathrm{s}$ up to a maximum frequency of $3.2459 \mathrm{rad} / \mathrm{s}$ [14]. The surface elevation is then calculated as

$$
\eta(t)=\sum_{i=1}^{100} \alpha(i) \sin (\omega(i) t+\phi(i))
$$

where

$$
\alpha(i)=\sqrt{2 E_{\omega}(\omega(i)) \Delta \omega}
$$

and $\phi(i)$ are random phase angles distributed uniformly from 0 to $2 \pi$.

\section{B. WEC model}

This paper considers a WEC of the heaving buoy type, which is an off-shore floating device that captures energy from its wave induced motion via hydraulic rams and a high pressure power take-off system. The WEC model is based on the equation of motion:

$$
\begin{gathered}
\ddot{x}(t)=\frac{1}{m_{b}+m_{r}(\infty)}\left\{\int_{-\infty}^{\infty} \eta(\tau) f(t-\tau) d \tau-B(t) \dot{x}(t)-\right. \\
\left.\int_{-\infty}^{t} k(t-\tau) \dot{x}(\tau) d \tau-R_{f} \dot{x}(t)-S x(t)\right\}
\end{gathered}
$$

where $\ddot{x}$ is the vertical acceleration of the buoy, $\dot{x}$ is its vertical velocity, $\eta(t)$ is the surface elevation, $B(t)$ is a nonlinear damping term representing the PTO system, $R_{f}$ is a resistance coefficient representing friction, $m_{r}(\infty)$ is the value of the added mass at infinite frequency, $m_{b}$ is the ballast mass, $S$ is the hydrostatic stiffness coefficient, $f(t)$ is the impulse response of the transfer function relating $\eta(t)$ and the excitation force $f_{e}(t)$ acting on the buoy, and $k(t)$ is the impulse response of the transfer function relating radiation damping to heave velocity. See [14] for an extensive discussion of this.

\section{Power Take-off (PTO) model}

The PTO used here is a hydraulic one that converts wave energy into water pressure needed for the desalination process and is modelled by

$$
\dot{P}_{\text {ro }}=-P_{\text {ro }}\left[\frac{1}{C}\left(\frac{1}{R_{t v}}+\frac{1}{R_{r o}}\right)\right]+\frac{|\dot{x}| A_{p}}{C}
$$


where $P_{r_{o}}$ is the pressure in the RO unit, $A_{p}$ is the pump area and

$$
\begin{aligned}
R_{t v} & =\frac{200 \sqrt{P_{r o}}}{C_{r}} \\
R_{r o} & =\frac{P_{r o}}{N_{r o} \rho_{r o}\left(P_{r o}-P_{o s m}\right)}
\end{aligned}
$$

$C$ is the accumulator, $C_{r}$ is the rated valve flow coefficient, $R_{t v}$ represents the throttle valve resistance, $R_{r o}$ represents the RO membrane resistance, and $N_{\text {ro }}$ is the number of RO units deployed.

\section{State-space representation}

The mechanical model of the buoy and the PTO model of the buoy are coupled and, around a desired equilibrium pressure of $P_{r o}=\overline{P_{r o}}=6 \cdot 10^{6} \mathrm{~Pa}$, can be represented by the state-space system as

$$
\begin{aligned}
{\left[\begin{array}{l}
\dot{x}_{1} \\
\dot{x}_{2} \\
\dot{x}_{3}
\end{array}\right]=} & {\left[\begin{array}{ccc}
0 & 1 & 0 \\
-\frac{S}{M} & -\frac{R_{f}+R_{c}}{M} & -\frac{\mathbf{A}_{\mathrm{p}}^{*}}{M} \\
0 & \frac{\mathbf{A}_{\mathrm{p}}^{*}}{C} & -\frac{\left(\overline{P_{r o}}-P_{\text {osm }}\right) p_{r o}}{C \cdot P_{r o}}
\end{array}\right]\left[\begin{array}{l}
x_{1} \\
x_{2} \\
x_{3}
\end{array}\right] } \\
& +\left[\begin{array}{c}
0 \\
0 \\
\frac{-C_{r} \sqrt{\overline{P_{r o}}}}{200 C}
\end{array}\right] u+\left[\begin{array}{c}
0 \\
\frac{1}{M} \\
0
\end{array}\right] f_{e}(t)
\end{aligned}
$$

where $\left[\begin{array}{lll}x_{1} & x_{2} & x_{3}\end{array}\right]^{T}=\left[\begin{array}{lll}x & \dot{x} & P_{r_{o}}\end{array}\right]^{T}$ are the states of the system ( $x$ is the displacement of the body from rest and $\dot{x}$ the vertical velocity of the buoy) and $u \in[0,100] \%$ is the control signal, which corresponds to a linearised valve characteristic as described in [14], [1]. The model (8) is nonlinear, since the switching term $\mathbf{A}_{\mathbf{p}}^{*}$ carries the sign of $\dot{x}_{2}$, that is

$$
\begin{aligned}
\mathbf{A}_{\mathbf{p}}^{*} & =\operatorname{sign}\left(x_{2}\right) A_{p} \\
& = \begin{cases}A_{p}, & \text { if } x_{2}>0 \\
0, & \text { if } x_{2}=0 \\
-A_{p}, & \text { if } x_{2}<0\end{cases}
\end{aligned}
$$

where $A_{p}$ is the pump area as defined earlier. In the above model, the kernel $k(t)$ has been approximated by the constant $R_{c}$.

\section{E. Controller}

The controller suggested in [14], [1] was a type of nonlinear PI controller which will be described in more detail in the proceeding sections. The general idea behind the design appears to have been (quite logically) to decouple the mechanical system from the PTO system and bestow good tracking and disturbance rejection properties on the PTO subsystem in order to achieve satisfactory pressure regulation. In this paper, we consider a more generic type of controller than suggested in [14], [1] for the stability proof, although the inspiration for this analysis was taken from that particular controller.
TABLE I

PARAMETERS USED FOR DESIGN AND SIMULATION

\begin{tabular}{|c|c|c|}
\hline$m_{b}$ & mass of the buoy & $9700 \mathrm{Kg}$ \\
\hline$m_{r}(\infty)$ & added mass at infinite frequency & $8700 \mathrm{Kg}$ \\
\hline$S$ & hydrostatic stiffness of the buoy & $86.4 \mathrm{kN} / \mathrm{m}$ \\
\hline$R_{f}$ & friction resistance coefficient & $200 \mathrm{Kg}$ \\
\hline$A_{p}$ & pump area & $0.1 \mathrm{~m}^{2}$ \\
\hline$p_{o}$ & accumulator pre-charge pressure & $2.5 \cdot 10^{6} P_{a}$ \\
\hline$\gamma$ & ratio of specific heats of gas at & 1.4 \\
\hline constant temperature and volume & accumulator capacitance & $\begin{array}{c}2.464 \cdot 10^{-7} \\
m^{3} / P_{a}\end{array}$ \\
\hline$P_{o s m}$ & RO osmotic pressure & $27 \cdot 10^{5} P_{a}$ \\
\hline$\rho_{r o}$ & RO permeability coefficient \\
(single unit) & $\begin{array}{c}1.2121 \cdot 10^{-10} \\
m^{3} / \mathrm{s} / P_{a}\end{array}$ \\
\hline$C_{r}$ & Rated valve flow coefficient & $\begin{array}{c}1.1397 \cdot 10^{-5} \\
m^{3} P_{a}^{0.5} / \mathrm{s}\end{array}$ \\
\hline$R_{c}$ & Static approximation of kernel & 722.1 \\
\hline
\end{tabular}

\section{STABILITY ANALYSIS}

\section{A. Plant model}

Equation (8) can be more concisely written as

$$
\begin{aligned}
{\left[\begin{array}{c}
\dot{x}_{1} \\
\dot{x}_{2} \\
\hline \dot{x}_{3}
\end{array}\right] } & =\left[\begin{array}{cc|c}
0 & 1 & 0 \\
A_{21} & A_{22} & -\frac{\mathbf{A}_{\mathbf{p}}^{*}}{M} \\
\hline 0 & \frac{\mathbf{A}_{\mathbf{p}}^{*}}{C} & A_{33}
\end{array}\right]\left[\begin{array}{l}
x_{1} \\
x_{2} \\
\frac{x_{3}}{C}
\end{array}\right] \\
& +\left[\begin{array}{c}
0 \\
\frac{1}{M} \\
\hline 0
\end{array}\right] f_{e}(t)+\left[\begin{array}{c}
0 \\
0 \\
\hline B_{3}
\end{array}\right] u \\
y & =\left[\begin{array}{lll}
0 & 0 & 1
\end{array}\right]\left[\begin{array}{c}
x_{1} \\
\frac{x_{2}}{x_{3}}
\end{array}\right]
\end{aligned}
$$

This model can be compactly written as

$$
\begin{aligned}
\dot{x} & =A x+A_{s}(x) x+B_{1} f_{e}(t)+B_{2} u \\
y & =C_{y} x
\end{aligned}
$$

where

$$
\begin{aligned}
A & =\left[\begin{array}{cc|c}
0 & 1 & 0 \\
A_{21} & A_{22} & 0 \\
\hline 0 & 0 & A_{33}
\end{array}\right] \\
A_{s}(x) & =\left[\begin{array}{ccc}
0 & 0 & 0 \\
0 & 0 & -\frac{A_{p}}{M} \operatorname{sign}\left(x_{2}\right) \\
0 & \frac{A_{p}}{C} \operatorname{sign}\left(x_{2}\right) & 0
\end{array}\right] \\
B_{1} & =\left[\begin{array}{c}
0 \\
\frac{1}{M} \\
\hline 0
\end{array}\right] \quad B_{2}=\left[\begin{array}{c}
0 \\
0 \\
\hline B_{3}
\end{array}\right] \quad C_{y}=\left[\begin{array}{lll}
0 & 0 & 1
\end{array}\right]
\end{aligned}
$$

Note that, disregarding the nonlinear terms, the system can be decoupled into two independent subsystems: an $\left(x_{1}, x_{2}\right)$ subsystem driven by the disturbance $f_{e}(t)$ and an $x_{3}$ subsystem driven by the control input $u(t)$. Also, the states $\left(x_{1}, x_{2}\right)$ are unobservable from the output $y(t)$. 


\section{B. A generic controller}

With the above observations in mind, a natural control strategy is one of decoupling the two subsystems. While complete decoupling is not possible, it is possible to use a pseudo-NDI type of control strategy to cancel the influence of the $\left(x_{1}, x_{2}\right)$ dynamics on the $x_{3}$ subsystem. Therefore, consider the following controller

$$
\mathcal{K} \sim\left\{\begin{array}{l}
\dot{x}_{c}=A_{c} x_{c}+B_{c r} r+B_{c y} y \\
u=C_{c} x_{c}+D_{c r} r+D_{c y} y+K_{s}(x)
\end{array}\right.
$$

where the nonlinear term $K_{s}(x)$ is chosen to "cancel" the nonlinear terms appearing in the $\dot{x}_{3}$ state equation, viz:

$$
\begin{aligned}
K_{s}(x) & =-\frac{1}{B_{3}} \frac{A_{p}}{C} \operatorname{sign}\left(x_{2}\right) x_{2} \\
& =-\frac{1}{B_{3}} \frac{A_{p}}{C}\left|x_{2}\right|
\end{aligned}
$$

Using the controller $\mathcal{K}$ then yields the following expression for the dynamics of the $\left(x_{3}, x_{c}\right)$ subsystem

$$
\begin{gathered}
{\left[\begin{array}{c}
\dot{x}_{3} \\
\dot{x}_{c}
\end{array}\right]=\underbrace{\left[\begin{array}{cc}
A_{33}+B_{3} D_{c y} & B_{3} C_{c} \\
B_{c y} & A_{c}
\end{array}\right]}_{A_{3, c}}\left[\begin{array}{l}
x_{3} \\
x_{c}
\end{array}\right]+} \\
\underbrace{\left[\begin{array}{c}
B_{3} D_{c r} \\
B_{c r}
\end{array}\right]}_{B_{3, c}} r
\end{gathered}
$$

Note that these dynamics are completely independent of the $\left(x_{1}, x_{2}\right)$ subsystem and that for this subsystem to be stable it is necessary that the controller $\mathcal{K}$ to be designed to stabilise the $\left(x_{3}, x_{c}\right)$ dynamics. This can be done using any standard linear method providing $K_{s}(x)$ is chosen as indicated in equation (15). Similarly, the dynamics of the $\left(x_{1}, x_{2}\right)$ subsystem read

$$
\begin{aligned}
{\left[\begin{array}{c}
\dot{x}_{1} \\
\dot{x}_{2}
\end{array}\right]=} & \underbrace{\left[\begin{array}{cc}
0 & 1 \\
A_{21} & A_{22}
\end{array}\right]}_{A_{1,2}}\left[\begin{array}{c}
x_{1} \\
x_{2}
\end{array}\right]+\underbrace{\left[\begin{array}{c}
0 \\
\frac{1}{M}
\end{array}\right]}_{B_{f}} f_{e}(t) \\
& +\underbrace{\left[\begin{array}{c}
0 \\
-\frac{A_{p}}{M} \operatorname{sign}\left(x_{2}\right)
\end{array}\right]}_{B_{1,2}\left(x_{2}\right)} x_{3}
\end{aligned}
$$

Thus defining $x_{1,2}:=\left[\begin{array}{ll}x_{1} & x_{2}\end{array}\right]^{\prime}$ and $x_{3, c}:=\left[\begin{array}{ll}x_{3} & x_{c}\end{array}\right]^{\prime}$ the dynamics of the complete system can be written as

$$
\begin{aligned}
{\left[\begin{array}{c}
\dot{x}_{1,2} \\
\dot{x}_{3, c}
\end{array}\right]=} & {\left[\begin{array}{cc}
A_{1,2} & B_{1,2}\left(x_{2}\right) \\
0 & A_{3, c}
\end{array}\right]\left[\begin{array}{l}
x_{1,2} \\
x_{3, c}
\end{array}\right]+\left[\begin{array}{c}
B_{f} \\
0
\end{array}\right] f_{e}(t) } \\
& +\left[\begin{array}{c}
0 \\
B_{3, c}
\end{array}\right] r
\end{aligned}
$$

\section{A Lyapunov analysis}

From the state-space realisation (18), it is clear that the $x_{3, c}$ dynamics are excited by the reference $r$ and not the $x_{1,2}$ dynamics. The $x_{1,2}$ dynamics are dependent on the $x_{3, c}$ dynamics but is useful to observe that

- $A_{1,2}$ is Hurwitz
- $A_{3, c}$ is Hurwitz (assuming the controller has been designed appropriately)

- The discontinuous term $B_{1,2}\left(x_{1,2}\right)$ is bounded, i.e. $\left\|B_{1,2}\left(x_{1,2}\right)\right\| \leq \beta:=A_{p} / M$

Thus the controller $\mathcal{K}$ provides a cascade system in which the two sub-systems are themselves asymptotically stable. It is now straightforward to prove internal stability of (18). Assuming that $f_{e}(t)=r(t)=0$ we choose our Lyapunov function as the sum of two quadratic Lyapunov functions associated with each sub-system, viz:

$$
V(x)=x_{1,2}^{\prime} P_{12} x_{1,2}+\alpha x_{3, c}^{\prime} P_{3 c} x_{3, c}
$$

where the matrices $P_{12}$ and $P_{3 c}$ are positive definite and $\alpha>0$ is a free scalar parameter. The time derivative of this Lyapunov function is

$$
\begin{aligned}
\dot{V}(x)= & 2 x_{1,2}^{\prime} P_{12}\left[A_{1,2} x_{1,2}+B_{1,2}\left(x_{1,2}\right) x_{3, c}\right]+ \\
& 2 \alpha x_{3, c}^{\prime} P_{3 c} A_{3, c} x_{3, c} \\
= & x_{1,2}^{\prime}\left[P_{12} A_{1,2}+A_{1,2}^{\prime} P_{12}\right] x_{1,2}+2 x_{1,2}^{\prime} P_{1,2} \\
& B_{1,2}\left(x_{1,2}\right) x_{3, c}+\alpha x_{3, c}^{\prime}\left[P_{3 c} A_{3, c}+A_{3, c}^{\prime} P_{3 c}\right] x_{3, c} \\
\leq & -x_{1,2}^{\prime} Q_{12} x_{1,2}-\alpha x_{3, c}^{\prime} Q_{3 c} x_{3 c} \\
& +2\left\|x_{1,2}\right\|\left\|P_{12}\right\|\left\|B_{1,2}\left(x_{1,2}\right)\right\|\left\|x_{3, c}\right\|
\end{aligned}
$$

where, since $A_{1,2}$ and $A_{3, c}$ are Hurwitz, $Q_{12}>0$ and $Q_{3 c}>$ 0 are solutions to the Lyapunov equations

$$
\begin{aligned}
P_{12} A_{1,2}+A_{1,2}^{\prime} P_{12} & =-Q_{12} \\
P_{3 c} A_{3, c}+A_{3, c}^{\prime} P_{3 c} & =-Q_{3 c}
\end{aligned}
$$

Also, noting that $\left\|B_{1,2}\left(x_{1,2}\right)\right\| \leq \beta$, enables us to write

$$
\begin{aligned}
V(x) \leq & x_{1,2}^{\prime} Q_{12} x_{1,2}-\alpha x_{3, c}^{\prime} Q_{3 c} x_{3, c}+2\left\|x_{1,2}\right\|\left\|x_{3, c}\right\| \beta\left\|P_{12}\right\| \\
\leq & \lambda_{\min }\left(Q_{12}\right)\left\|x_{1,2}\right\|^{2}-\alpha \lambda_{\min }\left(Q_{3 c}\right)\left\|x_{3, c}\right\|^{2} \\
& +2\left\|x_{1,2}\right\|\left\|x_{3, c}\right\| \beta\left\|P_{12}\right\| \\
= & {\left[\begin{array}{cc}
\left\|x_{1,2}\right\| \\
\left\|x_{3, c}\right\|
\end{array}\right] } \\
& {\left[\begin{array}{cc}
-\lambda_{\min }\left(Q_{12}\right) & \beta\left\|P_{12}\right\| \\
* & -\alpha \lambda_{\min }\left(Q_{3, c}\right)
\end{array}\right]\left[\begin{array}{c}
\left\|x_{1,2}\right\| \\
\left\|x_{3, c}\right\|
\end{array}\right] }
\end{aligned}
$$

It is always possible to choose a large enough $\alpha$ so that this inequality is negative definite, which thus implies the system is globally asymptotically stable. Note that this analysis is independent of the precise form of the controller $\mathcal{K}$ : all that is required is that it has the structure given in equation (13) and be such that the matrix $A_{3, c}$ be Hurwitz (which can be done using standard linear design techniques).

Remark: It is interesting to observe that the controller given in [1], [14] was a special form of this controller with state-space representation

$$
\mathcal{K}_{N R} \sim\left\{\begin{aligned}
\dot{x}_{c} & =-x_{3}+r \\
u & =k_{i} x_{c}-k_{p} x_{3}+\left(k_{p}+k_{f f}\right) r+K_{s}(x)
\end{aligned}\right.
$$


where $K_{s}(x)$ is given by equation (15); $k_{f f}=\frac{-A_{33}}{B_{3}}, k_{p}=$ $\frac{-\left(\mu_{1}+\mu_{2}\right)+A_{33}}{B_{3}}, k_{i}=\frac{\mu_{1} \mu_{2}}{B_{3}}$ are the feedforward and PI gains, respectively; and $\mu_{1}$ and $\mu_{2}$ are the closed-loop poles of the $x_{3, c}$ subsystem in (16). A block diagram of this controller is shown in Fig. 1. In [14] a stability analysis based on writing the system as a switched linear system and then searching for a common Lyapunov function was used. While this technique was able to prove open-loop stability, no conclusions about closed-loop stability could be made, and it was not obvious that this was easily inferred. Here we have shown that a generic class of pseudo-NDI controllers provide closed-loop global asymptotic stability, and, as the controller of [14] is a member of this class, that the control strategy adopted in [14] is globally asymptotically stabilising.

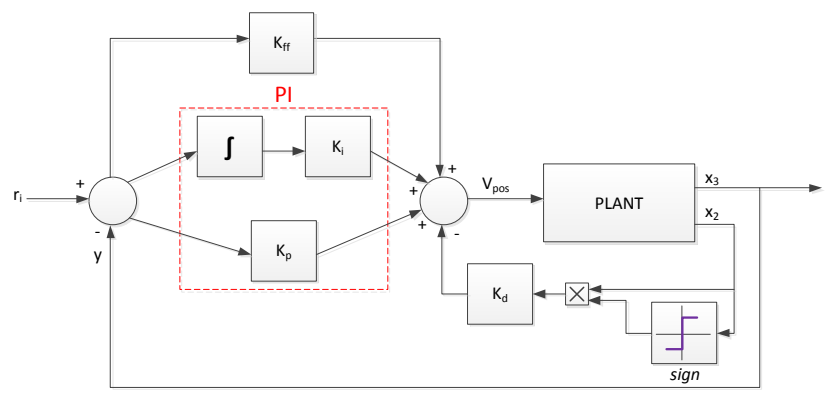

Fig. 1. Block diagram of the state feedback controller proposed in [1]

\section{CONCLUSIONS}

This paper has analysed a class of pseudo-NDI controllers for the production of potable water from a type of WEC. It has been proven that there is a family of controllers which stabilise this system; moreover this family of controllers simply requires one nonlinear "cancellation" term with the linear part of controller free to be constructed using standard linear design methods. The controller introduced in [1] falls into this category and thus allows one to conclude that the controller used there globally stabilises the system; only open-loop stability was hitherto known. Work currently underway is investigating the use of anti-windup compensators in accommodating control signal saturation in WEC control; results will be reported in due course.

\section{REFERENCES}

[1] G. Nolan and J. Ringwood, "Control of a Heaving Buoy Wave Energy Converter for Potable Water Production," Irish Signal and Systems Conference, Dublin, June 28-30 2006.

[2] P. Girard, "Pour divers moyens d'employer les vagues de la mer, comme moteurs." French Patent, July 12, 1799.

[3] J. Ringwood, "The dynamics of wave energy," Irish Signal and Systems Conference, Dublin, no. CP519, pp. 23-34, 28-30 June 2006.

[4] T. Brekken, B. Batten, and E. Amon, "From Blue to Green [Ask the Experts]," IEEE Control Systems Magazine, vol. 31, no. 5, pp. 18-24, October 2011.

[5] M. Santos, E. Tedeschi, P. Ricci, M. Molinas, and J. L. Martin, "Grid Connection Improvements by Control Strategy Selection for Wave Energy Converters," International Conference On Renewable Energy and Power Quality (ICREPQ11), Las Palmas de Gran Canaria, Spain, April 2011.
[6] F. Fusco and J. Ringwood, "Suboptimal Causal Reactive Control of Wave Energy Converters Using a Second Order System Model," in 21 st International Offshore (Ocean) and Polar Engineering Conference (ISOPE), Maui, USA, June 2011, pp. 687-694.

[7] A. G. Santana, D. E. M. Andade, and A. D. L. V. Jaen, "Output power of linear generators under reactive control in regular waves," International Conference On Renewable Energy and Power Quality (ICREPQ11), Las Palmas de Gran Canaria, Spain, 2011.

[8] F. Fusco and J. V. Ringwood, "A Study of the Prediction Requirements in Real-Time Control of Wave Energy Converters," IEEE Transactions on Sustainable Energy, vol. 3, no. 1, January 2012.

[9] J. Ringwood and S. Butler, "Optimisation of a wave energy converter," in Proc. IFAC Conference On Control Applications in Marine Systems, Ancona, Italy, July 2004.

[10] J. Hals, "Modelling and phase control of wave-energy converters," Ph.D. dissertation, Norwegian University of Science and Technology, May 2010.

[11] T. K. A. Brekken, "On Model Predictive Control for a point absorber Wave Energy Converter," in IEEE PowerTech, Trondheim, Norway, June 2011, pp. 1-8.

[12] J. A. M. Cretel, G. Lightboy, G. P. Thomas, and A. W. Lewis, "Maximisation of Energy Capture by a Wave-Energy Point Absorber using Model Predictive Control," 18th IFAC World Congress, Milano, Italy, September 2011.

[13] K. Schlemmer, F. Fuchshumer, N. Bhmer, R.Costello, and C. Villegas, "Design and control of a hydraulic power take-off for an axi-symmetric heaving point absorber," in Proc. 9th European Wave and Tidal Energy Conference (EWTEC), 2011.

[14] G. A. Nolan, "Modelling and Optimisation of a Heaving Buoy Wave Energy Converter for Potable Water Production," Ph.D. dissertation, National University of Ireland, Maynooth, July 2006.

[15] D. Magagna, D. Stagonas, D. Warbrick, and G. Muller, "Resonating wave energy converter for delivery of water for desalination and energy generation," 8th European Wave and Tidal Energy, Uppsala, Sweden, 2009.

[16] G. Bacelli, J.-C. Gilloteaux, and J. Ringwood, "A predictive controller for a heaving buoy producing potable water," in Proceedings of the European Control Conference, ECC09,, Budapest, Hungary, 23-26 August 2009.

[17] W. J. J. Pierson and L. Moskowitz, "A Proposed Spectral Form for Fully Developed Wind Seas Based on the Similarity Theory of S. A Kitaigorodskii," Journal of Geophysical Research, vol. 69, no. 24, pp. 5181-5190, 1964. 